University of Warwick institutional repository: http://go.warwick.ac.uk/wrap This paper is made available online in accordance with publisher policies. Please scroll down to view the document itself. Please refer to the repository record for this item and our policy information available from the repository home page for further information.

To see the final version of this paper please visit the publisher's website. Access to the published version may require a subscription.

Author(s): D. COLLIER and I. D. MORRIS

Article Title: Approximating the maximum ergodic average via periodic orbits

Year of publication: 2008

Link to published version:

http://dx.doi.org/10.1017/S014338570700082X

Publisher statement: None 


\title{
Approximating the maximum ergodic average via periodic orbits
}

\author{
D. COLLIER $\dagger$ and I. D. MORRIS $\ddagger$ \\ $\dagger$ School of Mathematics, University of Manchester, Manchester M13 9PL, UK \\ (e-mail:dcollier@maths.manchester.ac.uk) \\ $\$$ Mathematics Institute, University of Warwick, Coventry CV4 7AL, UK \\ (e-mail:Ian.Morris@warwick.ac.uk)
}

(Received 9 February 2006 and accepted in revised form 4 September 2007)

Abstract. Let $\sigma: \Sigma_{A} \rightarrow \Sigma_{A}$ be a subshift of finite type, let $\mathcal{M}_{\sigma}$ be the set of all $\sigma$-invariant Borel probability measures on $\Sigma_{A}$, and let $f: \Sigma_{A} \rightarrow \mathbb{R}$ be a Hölder continuous observable. There exists at least one $\sigma$-invariant measure $\mu$ which maximizes $\int f d \mu$. The following question was asked by B. R. Hunt, E. Ott and G. Yuan: how quickly can the maximum of the integrals $\int f d \mu$ be approximated by averages along periodic orbits of period less than $p$ ? We give an example of a Hölder observable $f$ for which this rate of approximation is slower than stretched-exponential in $p$.

\section{Introduction}

Let $\sigma: \Sigma_{A} \rightarrow \Sigma_{A}$ be a subshift of finite type, let $\mathcal{M}_{\sigma}$ be the set of all $\sigma$-invariant Borel probability measures on $\Sigma_{A}$, and let $f: \Sigma_{A} \rightarrow \mathbb{R}$ be Hölder continuous. There is at least one invariant measure $\mu \in \mathcal{M}_{\sigma}$, which we term a maximizing measure for $f$, such that

$$
\int f d \mu=\beta(f):=\sup _{\nu \in \mathcal{M}_{\sigma}} \int f d \nu .
$$

In this paper we investigate the problem of finding invariant measures supported on periodic orbits which approximately realize this maximum. More specifically, if we let $\mathcal{M}_{\sigma, p}$ be the the set of all ergodic $\sigma$-invariant measures supported on points fixed by $\sigma^{p}$, we investigate the quantity

$$
E_{f}(p)=\sup _{\nu \in \mathcal{M}_{\sigma}} \int f d \nu-\sup _{\nu \in \mathcal{M}_{\sigma}, p} \int f d \nu,
$$

introduced by Yuan and Hunt [11], which is the difference between the maximum integral $\beta(f)$ of $f$ and the 'best approximating' periodic orbit whose period divides $p$.

Our motivation is twofold. Firstly, the above problem is of interest in control theory: if we are able to control the dynamical system $\sigma: \Sigma_{A} \rightarrow \Sigma_{A}$, we will wish to direct the trajectories of points in $\Sigma_{A}$ toward particular unstable orbits which optimize some given quantity. For reasons of computational simplicity, it is desirable that such orbits should be periodic and have small length. This leads us to ask how close to optimal orbits of 
a specified period may be. These considerations motivate the work of Yuan, Hunt and Ott $[7,11]$.

Secondly, the quantity $\beta(f)$ has shown itself to be of interest in a number of situations in ergodic theory, both intrinsically $[\mathbf{1 , 2}, \mathbf{6}, \mathbf{8}]$ and in application to existing problems $[\mathbf{3 , 4}, \mathbf{9}]$. It is therefore of interest to be able to compute this quantity accurately in numerical experiments. One obvious approach to this task would be to exhaustively compute ergodic averages of $f$ along periodic orbits of length up to $n$, and take the supremum of these averages as an approximation to $\beta(f)$. The error incurred in this approximation would therefore equal $\inf _{1 \leq p \leq n} E_{p}(f)$.

It is a classical result [10] that $\bigcup_{p=1}^{\infty} \mathcal{M}_{\sigma, p}$ is dense in $\mathcal{M}_{\sigma}$ in the weak-* topology, and so $E_{f}(p) \rightarrow 0$ as $p \rightarrow \infty$ for all continuous $f$. We wish to investigate the rate at which this convergence occurs when $f$ is Hölder.

The behaviour of $E_{f}(p)$ as $p \rightarrow \infty$ is at present poorly understood. On the strength of numerical experiments [7] combined with rigorous analysis, Yuan and Hunt [11] observed that the sequence $E_{f}(p)$ often decays to zero at an exponential rate, but were unable to prove this in generality. They then asked whether it could be shown that $E_{f}(p)$ always decays exponentially.

The purpose of this paper is to answer this question in the negative. We have the following theorem.

THEOREM 1. Let $\sigma: \Sigma_{A} \rightarrow \Sigma_{A}$ be a subshift of finite type. There exists Hölder continuous $f: \Sigma_{A} \rightarrow \mathbb{R}$ such that $E_{f}(p)$ tends to 0 at a slower than stretched-exponential rate: $\log E_{f}(p)=o\left(p^{\varepsilon}\right)$ for every $\varepsilon>0$.

We note that a result similar to Theorem 1 has recently been proved by Bressaud and Quas in [5], in which the quantity $\inf _{1 \leq p \leq n} E_{p}(f)$ is considered. Bressaud and Quas were able to obtain upper and lower bounds that are superior to those in the present article, but with the weakness that their lower bound applies only along subsequences of integers $n$.

\section{Notation and definitions}

Let $A$ be an irreducible aperiodic $N \times N$ matrix of zeros and ones. We define the shift space associated with $A$ to be the set

$$
\Sigma_{A}:=\left\{x=\left(x_{i}\right)_{i \geq 1}: x_{i} \in\{1, \ldots, N\} \text { and } A\left(x_{i}, x_{i+1}\right)=1 \text { for all } i \geq 1\right\},
$$

and define the shift map $\sigma: \Sigma_{A} \rightarrow \Sigma_{A}$ by

$$
(\sigma x)_{i}=x_{i+1}
$$

for all $i \geq 1$. Given $\theta \in(0,1)$, we define the $\theta$-metric $d_{\theta}$ on $\Sigma_{A}$ by

$$
d_{\theta}\left(\left(x_{i}\right)_{i \geq 1},\left(y_{i}\right)_{i \geq 1}\right)=\theta^{\inf \left\{n \geq 1: x_{n} \neq y_{n}\right\}} .
$$

We say that a function $f: \Sigma_{A} \rightarrow \mathbb{R}$ is $\theta$-Hölder continuous if it is Lipschitz continuous with respect to the metric $d_{\theta}$. We fix $\theta \in(0,1)$ for the remainder of this paper.

We define a finite word to be a finite sequence $\omega=\left(\omega_{i}\right)_{i=1}^{n}$ taking values in the set $\{1,2, \ldots, N\}$. We say that $\omega=\left(\omega_{i}\right)_{i=1}^{n}$ is compatible with the matrix $A$ if $A\left(\omega_{i}, \omega_{i+1}\right)=1$ for all $i<n$. We define the length of the word $(\omega)_{i=1}^{n}$ to be $n$. We will on occasion describe elements of $\Sigma_{A}$ as infinite words. We say that finite words $\omega^{1}, \omega^{2}$ of 
equal length $n$ are rotation equivalent if there exists a non-negative integer $r<n$ such that $\omega_{i}^{1}=\omega_{i+r}^{2}$ whenever $1 \leq i \leq n-r$, and $\omega_{i}^{1}=\omega_{i+r-n}^{2}$ whenever $n-r<i \leq n$. When $\omega^{1}$ and $\omega^{2}$ are rotation equivalent we write $\omega^{1} \simeq \omega^{2}$. Words of distinct lengths are never rotation equivalent.

Given two words $\omega^{1}$ and $\omega^{2}$ with lengths $n_{1}$ and $n_{2}$, respectively, we define their concatenation $\omega^{1} \cdot \omega^{2}$ to be the word of length $n_{1}+n_{2}$ given by $\left[\omega^{1} \cdot \omega^{2}\right]_{i}=\omega_{i}^{1}$ when $1 \leq i \leq n_{1}$, and $\left[\omega^{1} \cdot \omega^{2}\right]_{i}=\omega_{i}^{2}$ when $n_{1}+1 \leq i \leq n_{1}+n_{2}$. Given a finite list of words $\omega^{1}, \ldots, \omega^{m}$, we denote the compound concatenation $\omega^{1} \cdot \omega^{2} \cdots \omega^{m}$ by $\prod_{k=1}^{m} \omega^{k}$. Concatenation is associative. If $\omega^{1}, \omega^{2}$ are finite words with lengths $n_{1}, n_{2}$ compatible with $A$, their concatenation is compatible with $A$ if and only if $A\left(\omega_{n_{1}}^{1}, \omega_{1}^{2}\right)=1$.

For each $p>0$, we let $\Omega_{p}$ be the set of all words of length $p$ which are compatible with $A$. We let $\bar{\Omega}_{p}$ be the set of all words $\omega \in \Omega_{p}$ such that $A\left(\omega_{p}, \omega_{1}\right)=1$. Note that $\omega \in \bar{\Omega}_{p}$ if and only if both $\omega \in \Omega_{p}$ and $\omega \cdot \omega \in \Omega_{2 p}$.

If $x \in \Sigma_{A}$, it is clear that $\sigma^{p} x=x$ if and only if $x_{i}=x_{i+p}$ for all $i \geq 1$, if and only if there exists $\omega \in \bar{\Omega}_{p}$ such that $x_{i+k p}=\omega_{i}$ for all $1 \leq i \leq p$ and $k \geq 0$. In this case we write $x=\pi(\omega)$. This defines a relationship between the sets Fix $p=\left\{x \in \Sigma_{A}: \sigma^{p} x=x\right\}$ and $\bar{\Omega}_{p}$, which is readily seen to be bijective. Moreover, we have $\pi\left(\omega^{1}\right)=\sigma^{j} \pi\left(\omega^{2}\right)$ for some $j \geq 0$ if and only if $\omega^{1} \simeq \omega^{2}$.

If $a=\left(a_{i}\right)_{i=1}^{n}$ is a finite word and $b$ is either a finite word or an element of $\Sigma_{A}$, we write $a \prec b$ if there is $k \geq 0$ such that $a_{i}=b_{i+k}$ for all $1 \leq i \leq n$. In this case we say that $a$ is a subword of $b$.

\section{Proof of Theorem 1}

We begin with the following.

Proposition 3.1. Let $K=\sigma K$ be a closed non-empty subset of $\Sigma_{A}$, and define $f_{K}(x)=$ $-d_{\theta}(K, x)$ for all $x \in \Sigma_{A}$. Clearly $f_{K}$ is $\theta$-Hölder continuous. For each $\omega \in \Omega_{p}$ we define

$$
\xi(\omega, K):=\inf _{\omega^{\prime} \simeq \omega}\left\{\ell>0: \exists a \in \Omega_{\ell} \text { such that } a \prec \omega^{\prime} \text { and } \forall x \in K, a \nprec x\right\} .
$$

Let $p>0$, and suppose that $\sup _{\omega \in \Omega_{p}} \xi(\omega, K) \leq p$. Then

$$
E_{f_{K}}(p) \geq \frac{1}{p} \theta^{\sup _{\omega \in \Omega_{p}} \xi(\omega, K)}=\frac{1}{p} \inf _{\omega \in \Omega_{p}} \theta^{\xi(\omega, K)} .
$$

Proof. Let $p>0$ and $\omega \in \Omega_{p}$, where $\xi(\omega, K) \leq p$. We have

$$
\begin{aligned}
\log _{\theta} \sup _{\omega^{\prime} \simeq \omega} d\left(\pi\left(\omega^{\prime}\right), K\right)=\log _{\theta} \sup _{\omega^{\prime} \simeq \omega} \inf _{x \in K} d_{\theta}\left(\pi\left(\omega^{\prime}\right), x\right) \\
=\inf _{\omega^{\prime} \simeq \omega} \sup _{x \in K} \inf \left\{\ell>0: x_{\ell} \neq \pi\left(\omega^{\prime}\right)_{\ell}\right\} \\
=\inf _{\omega^{\prime} \simeq \omega} \sup \left\{\ell>0: \exists x \in K \text { such that } x_{i}=\pi\left(\omega^{\prime}\right)_{i} \forall 1 \leq i<\ell\right\} \\
=\inf _{\omega^{\prime} \simeq \omega}\left\{\ell>0: \exists a \in \Omega_{\ell} \text { such that } a_{i}=\pi\left(\omega^{\prime}\right)_{i} \forall 1 \leq i \leq \ell \text { and } \forall x \in K, a \nprec x\right\} \\
=\inf _{\omega^{\prime} \simeq \omega}\left\{\ell>0: \exists a \in \Omega_{\ell} \text { such that } a \prec \pi\left(\omega^{\prime}\right) \text { and } a \nprec x \forall x \in K\right\} \\
=\inf _{\omega^{\prime} \simeq \omega}\left\{\ell>0: \exists a \in \Omega_{\ell} \text { such that } a \prec \omega^{\prime} \text { and } a \nprec x \forall x \in K\right\} \\
=\xi(\omega, K),
\end{aligned}
$$


where we have used $\xi(\omega, K) \leq p$ in the second-from-last equality. Since $K$ is closed, $\sigma$-invariant and non-empty, the Krylov-Bogolioubov theorem shows that there exists $\mu \in \mathcal{M}_{\sigma}$ such that $\mu(K)=1$. It follows that $\beta(f)=0$, and so

$$
\begin{aligned}
E_{f_{K}}(p) & =\inf _{\sigma^{p} x=x} \frac{1}{p} \sum_{j=0}^{p-1} d\left(\sigma^{j} x, K\right) \\
& \geq \frac{1}{p} \inf _{\sigma^{p} x=x} \sup _{0 \leq j<p} d\left(\sigma^{j} x, K\right) \\
& =\frac{1}{p} \inf _{\omega \in \bar{\Omega}_{p}} \sup _{\omega^{\prime} \simeq \omega} d\left(\pi\left(\omega^{\prime}\right), K\right) \\
& \geq \frac{1}{p} \inf _{\omega \in \Omega_{p}} \theta^{\xi(\omega, K)},
\end{aligned}
$$

as required.

To prove Theorem 1, it therefore suffices to construct a non-empty compact set $K=\sigma K \subseteq \Sigma_{A}$ such that $\sup _{\omega \in \Omega_{p}} \xi(\omega, K)=o\left(p^{\varepsilon}\right)$ as $p \rightarrow \infty$. The remainder of this section is dedicated to this task. We will construct the invariant set $K$ recursively, using a sequence of sets of words $M_{n}$ of increasing length. The set $K$ will then arise as a limit of these sets.

Let $M_{1}$ be a subset of $\Omega_{\ell_{1}}$ for some positive integer $\ell_{1}$ such that $m_{1}=$ Card $M_{1}$ is divisible by $2^{16}$. We require that $M_{1}$ have the following properties.

Definition 3.2. There exists an integer $P \geq 1$ and a word $z=\left(z_{i}\right)_{i=1}^{P} \in \Omega_{P}$ such that:

(i) every $\omega \in M_{1}$ satisfies $z_{i}=\omega_{i}$ for all $1 \leq i \leq P$;

(ii) if $\omega=\omega^{1} \cdot \omega^{2}$ where $\omega^{1}, \omega^{2} \in M_{1}$, and $z_{i}=\omega_{i+r}$ for all $1 \leq i \leq P$, then either $r=\ell_{1}$ or $r=0$

(iii) $A\left(\omega_{\ell_{1}}^{1}, \omega_{1}^{2}\right)=1$ for all $\omega^{1}, \omega^{2} \in M_{1}$; that is, $\omega^{1} \cdot \omega^{2} \in \Omega_{2 \ell_{1}}$ for every pair $\omega^{1}$, $\omega^{2} \in M_{1}$.

The reader may verify that such a set $M_{1}$ can be constructed for any prescribed $m_{1}$ and matrix $A$.

A sequence of sets $M_{n}$ with cardinalities $m_{n}$ consisting of words of length $\ell_{n}$ will be defined in an inductive fashion, starting with the set $M_{1}$. We begin by introducing some integer sequences which will be crucial to our construction.

Definition 3.3. Given an integer $m_{1}$ divisible by $2^{16}$, let $q_{1}=t_{1}=m_{1} / 4$. Define sequences $\left(m_{n}\right)_{n \geq 1},\left(q_{n}\right)_{n \geq 1}$ and $\left(t_{n}\right)_{n \geq 1}$ as follows. Given the integer $t_{n}$, let $m_{n+1}=4^{t_{n}}$ and $q_{n+1}=(1 / 4) m_{n+1}=4^{t_{n}-1}$, and let $\tau_{n+1}$ be the unique positive real number such that

$$
\tau_{n+1}^{\sqrt{\tau_{n+1}}}=4^{t_{n}-1}=q_{n+1} .
$$

Then define

$$
t_{n+1}=4^{\left\lfloor\left(t_{n} / \sqrt{\tau_{n+1}}\right)\right\rfloor-1} .
$$

The key properties of these sequences are stated in the lemma below. 
LEMMA 3.4. For each $n \geq 1$, we have $t_{n} \mid q_{n}, m_{n} / q_{n}=4$ and $t_{n} \geq 2^{14}$. Moreover, the sequence $\left(t_{n}\right)_{n \geq 1}$ satisfies $t_{n} / \sqrt{t_{n+1}} \leq 16 t_{n}^{1 / 4}$ for all $n \geq 1$ and

$$
\lim _{n \rightarrow \infty} \frac{t_{n}}{t_{n+1}}=0
$$

Proof. The first two statements are clear. We consider the sequence $\left(t_{n}\right)_{n \geq 1}$; the definition implies that

$$
\frac{1}{16} \tau_{n+1}=4^{\left(t_{n}-1 / \sqrt{\tau_{n+1}}\right)-2} \leq 4^{\left\lfloor\left(t_{n} / \sqrt{\tau_{n+1}}\right)\right\rfloor-1}=t_{n+1} \leq 4^{\left(t_{n} / \sqrt{\tau_{n+1}}\right)-1} \leq \tau_{n+1},
$$

for every $n \geq 1$. We proceed inductively. Given that $t_{n} \geq 2^{14}$, notice that

$$
\sqrt{\tau_{n+1}} \log \tau_{n+1}=\left(t_{n}-1\right) \log 4 .
$$

We thus have

$$
t_{n}=\sqrt{\tau_{n+1}} \log _{4} \tau_{n+1}+1>\sqrt{\tau_{n+1}},
$$

and therefore

$$
\log _{4} \tau_{n+1}<2 \log _{4} t_{n}
$$

We deduce

$$
\sqrt{t_{n+1}} \geq \frac{1}{4} \sqrt{\tau_{n+1}}=\frac{t_{n}-1}{4 \log _{4} \tau_{n+1}} \geq \frac{t_{n}-1}{8 \log _{4} t_{n}} \geq \frac{1}{8} t_{n}^{3 / 4}-\frac{1}{8},
$$

where we have used the elementary inequality $\log _{4} t \leq t^{1 / 4}$ for all $t \geq 16$. Thus

$$
t_{n+1} \geq \frac{1}{64}\left(t_{n}^{3 / 4}-1\right)^{2} \geq 2^{-6}\left(2^{21}-2^{11} \sqrt{2}+1\right)>2^{14},
$$

for every $n \geq 1$, making it clear that $t_{n} / \sqrt{t_{n+1}} \leq 16 t_{n}^{1 / 4}$. One may easily use the above to show that $t_{n+1} \geq t_{n}+1 / 64$ for each $n \geq 2$, which implies that $t_{n} \rightarrow \infty$; since for each $n$ we have

$$
0 \leq \frac{t_{n}}{t_{n+1}} \leq \frac{4 t_{n}}{t_{n}^{3 / 2}-2 t_{n}^{3 / 4}+1}
$$

it follows that $\lim _{n \rightarrow \infty} t_{n} / t_{n+1}=0$ as required.

For an integer $n \geq 1$, a finite word $a=\left(a_{i}\right)_{i=1}^{m}$ and a finite or infinite word $b$, we shall write $a \prec_{n} b$ if there is $k \geq 0$ such that $a_{i}=b_{i+k \ell_{n}}$ for all $1 \leq i \leq m$. The distinction between $\prec$ and $\prec_{n}$ will be important since we will construct words $\omega \in M_{n+1}$ as concatenations of words $a, b, c, \ldots \in M_{n}$. For example, if $a, b, c \in M_{n}$, then it is true that $b \cdot c \prec_{n} a \cdot b \cdot c \cdot a$ and $c \cdot b \nprec_{n} a \cdot b \cdot c \cdot a$; however, the statement $c \cdot b \prec a \cdot b \cdot c \cdot a$ could be either true or false, depending on the subword structure of the words $a, b$ and $c$.

Given $n \geq 1$ and the set $M_{n}$, we construct the set $M_{n+1}$ as follows. Recalling that $m_{n}=4 q_{n}$, partition $M_{n}$ into $q_{n}$ disjoint sets $C_{n}^{k}$ of cardinality 4 , where $1 \leq k \leq q_{n}$. For each $k$, we will write $C_{n}^{k}=\left\{c_{j}^{k}: 1 \leq j \leq 4\right\}$. Define

$$
I_{n}=\left\{\left(i_{1}, i_{2}, \ldots, i_{q_{n}}\right) \in\{1,2,3,4\}^{q_{n}}: i_{m}=i_{m+t_{n}} \text { for all } 1 \leq m \leq q_{n}-t_{n}\right\} .
$$

For each $q_{n}$-tuple $\left(i_{1}, \ldots, i_{t_{n}}\right) \in I_{n}$, we construct the word

$$
\omega_{\left(i_{1}, \ldots, i_{q_{n}}\right)}:=\prod_{k=1}^{q_{n}} c_{i_{k}}^{k} .
$$


Let $M_{n+1}=\left\{\omega_{\left(i_{1}, \ldots, i_{q_{n}}\right)}:\left(i_{1}, \ldots, i_{q_{n}}\right) \in I_{n}\right\}$, then clearly

$$
\text { Card } M_{n+1}=\operatorname{Card} I_{n}=4^{t_{n}}=m_{n+1},
$$

in accordance with Definition 3.4. We remark that Definition 3.2(iii) implies that $M_{n} \subseteq \Omega_{\ell_{n}}$ for every $n \geq 1$. The key features of the above construction are summarized in the following lemma.

LEMMA 3.5. The following are direct consequences of the definition of the sets $M_{n}$.

(i) If $\prod_{k=1}^{q_{n}} c_{j_{k}}^{k} \in M_{n}$, where $n \geq 2$ and each $c_{j_{k}}^{k} \in C_{n-1}^{k}$, then $c_{j_{k}}^{k} \in C_{n-1}^{1}$ if and only if $k=1$.

(ii) If $\omega_{1}=c_{j_{1}}^{k_{1}} \cdot c_{j_{2}}^{k_{2}}$ with $c_{j_{1}}^{k_{1}} \in C_{n}^{k_{1}}$ and $c_{j_{2}}^{k_{2}} \in C_{n}^{k_{2}}$, then there exists $N>n$ and $\omega_{2} \in M_{N}$ such that $\omega_{1} \prec_{n} \omega_{2}$ if and only if $k_{2}=1+k_{1} \bmod q_{n}$.

(iii) If $\omega_{1}=\prod_{k=k_{0}}^{k_{0}+t_{n}} c_{j_{k}}^{k}$, where each $c_{j}^{k} \in C_{n}^{k}$ and $k_{0} \leq q_{n}-t_{n}$ with $j_{k_{0}} \neq j_{k_{0}+t_{n}}$, then $\omega_{1} \nprec_{n} \omega_{2}$ for all $\omega_{2} \in M_{N}$ whenever $N \geq n+1$.

(iv) If $\omega_{1} \prec_{n} \omega_{2} \in M_{n+1}, \omega_{1} \prec_{n} \omega_{3} \in M_{n+1}$ and $\omega_{1}$ has length at least $t_{n} \ell_{n}$, then $\omega_{2}=\omega_{3}$.

(v) For each $n \geq 1$ we have

$$
\ell_{n+1}=q_{n} \ell_{n}=\frac{1}{4} m_{n} \ell_{n}
$$

and hence

$$
\ell_{n+1}=\ell_{1} \prod_{k=1}^{n} q_{k}
$$

The proof is clear.

The following lemma allows us to pass from the relation $\prec$ to the relation $\prec_{n}$, and thus make use of Lemma 3.5.

LEMMA 3.6. Let $a=\left(a_{i}\right)_{i=1}^{\ell_{n}} \in M_{n}$ and $\omega=\left(\omega_{i}\right)_{i=1}^{\ell_{N}} \in M_{N}$ where $n<N$. Suppose that there is $r \geq 0$ such that $a_{i}=\omega_{r+i}$ for all $1 \leq i \leq \ell_{n}$. Then $\ell_{n} \mid r$.

Proof. We first prove the case $n=1$. Let $\omega=\prod_{k=1}^{m} \omega^{k}$ where each $\omega^{k} \in M_{1}$, and suppose $a_{i}=\omega_{r+i}$ for all $1 \leq i \leq \ell_{1}$. There exists $k^{*}$ such that $k^{*} \ell_{1} \leq r+i<\left(k^{*}+2\right) \ell_{1}$ for all $1 \leq i \leq \ell_{1}$, so that if we let $\hat{\omega}=\omega^{k^{*}} \cdot \omega^{k^{*}+1}$, then $z_{i}=a_{i}=\hat{\omega}_{i+r-k^{*} \ell_{1}}$ for all $1 \leq i \leq P \leq \ell_{1}$ by Definition 3.2(i). By Definition 3.2(ii) we have either $r-k^{*} \ell_{1}=\ell_{1}$ or $r-k^{*} \ell_{1}=0$, and so $\ell_{1} \mid r$ as required.

We proceed by induction on $n$. Let $a=\prod_{k=1}^{q_{n-1}} \hat{a}^{k}$ and $\omega=\prod_{k=1}^{m} \hat{\omega}^{k}$, with each $\hat{a}^{k}$, $\hat{\omega}^{k} \in M_{n-1}$. Let $\hat{a}^{1}=\left(\hat{a}_{i}^{1}\right)_{i=1}^{\ell_{n-1}}$. Since $\hat{a}_{i}^{1}=\omega_{r+i}$ for all $1 \leq i \leq \ell_{n-1}$, we have $\ell_{n-1} \mid r$ by the induction hypothesis. Since each $\hat{\omega}^{k}$ has length $\ell_{n-1}$, it follows that there is $s=r / \ell_{n-1}>0$ such that $\prod_{k=1}^{q_{n-1}} \hat{a}^{k}=\prod_{k=s}^{k=s+q_{n-1}} \hat{\omega}^{k}$. By Lemma 3.5(i) we have $\hat{a}^{k} \in C_{n-1}^{1}$ if and only if $k=1$, and similarly $\hat{\omega}^{k} \in C_{n-1}^{1}$ if and only if $k \equiv 1 \bmod q_{n}$. Since $\hat{\omega}^{s+1} \in C_{n-1}^{1}$ it follows that $s \equiv 0 \bmod q_{n}$. Since $r=s \ell_{n-1}$ and $\ell_{n}=q_{n-1} \ell_{n-1}$, we deduce that $\ell_{n} \mid r$.

Henceforth, we shall say that a finite word $\omega$ is admissible if and only if it occurs as a subword of some $\omega_{n} \in M_{n}$ for some $n$ (and hence for all sufficiently large $n$ ). We deduce the following. 
COROLlARY 3.7. Let $\omega=\left(\omega_{i}\right)_{i=1}^{p}$ be admissible. Let $a, b \in M_{n}$ and write $a=\left(a_{i}\right)_{i=1}^{\ell_{n}}$, $b=\left(b_{i}\right)_{i=1}^{\ell_{n}}$. If there are $r, s>0$ such that $a_{i}=\omega_{i+r}, b_{i}=\omega_{i+s}$ for all $1 \leq i \leq \ell_{n}$, then $\ell_{n} \mid r-s$.

We may now prove the next lemma.

LEMMA 3.8. The following constraints on admissibility hold.

(i) If $\omega=c_{j_{1}}^{k_{1}} \cdot c_{j_{2}}^{k_{2}}$ with $c_{j_{1}}^{k_{1}} \in C_{n}^{k_{1}}$ and $c_{j_{2}}^{k_{2}} \in C_{n}^{k_{2}}$, then $\omega$ is admissible if and only if $k_{2}=1+k_{1} \bmod q_{n}$.

(ii) If $\omega=\prod_{k=k_{0}}^{k_{0}+t_{n}} c_{j_{k}}^{k}$ with each $c_{j_{k}}^{k} \in C_{n}^{k}$ and $j_{k_{0}} \neq j_{k_{0}+t_{n}}$ where $k_{0} \leq q_{n}-t_{n}$, then $\omega$ is not admissible.

Proof. Apply Lemma 3.5 and Lemma 3.6.

LeMma 3.9. Let $a, b \in M_{n}$ where $n \geq 2$, and suppose that the word $a \cdot b$ is inadmissible. Then $a \cdot b$ has an inadmissible subword of length less than or equal to $2 t_{n-1} \ell_{n-1}$.

Proof. Let $a=\prod_{k=1}^{q_{n-1}} a^{k}$ and $b=\prod_{k=1}^{q_{n-1}} b^{k}$ where each $a^{k}, b^{k} \in M_{n-1}$. Define $u=$ $\prod_{k=q_{n-1}-t_{n-1}+1}^{q_{n-1}} a^{k}$ and $v=\prod_{k=1}^{t_{n-1}} b^{k}$, each being an admissible word of length $t_{n-1} \ell_{n-1}$. Clearly $u \cdot v \prec a \cdot b$. Suppose, for a contradiction, that $u \cdot v \prec \omega_{1} \cdot \omega_{2}=\omega$, say, where $\omega_{1}, \omega_{2} \in M_{n}$ and $\omega$ is admissible. Let $(u \cdot v)_{i}=\omega_{i+r}$ for all $1 \leq i \leq 2 \ell_{n-1}$. By Lemma 3.6, $r=\ell_{n-1} s$ for some $s$. Suppose that $s \geq q_{n}$; then $v \prec_{n} \omega_{2}$ and so $\omega_{2}=b$ by Lemma 3.5(iv). Thus $s=q_{n}$ and hence $u \prec_{n} \omega_{1}$ and $\omega_{1}=a$. Therefore $\omega=a \cdot b$ and so is not admissible. It follows that $u \cdot v$ is inadmissible; this word has length $2 t_{n-1} \ell_{n-1}$ as required.

LEMMA 3.10. Let $\omega$ be a word of length $\ell \geq \ell_{n}$, where $n \geq 2$. Then either $\omega \simeq \prod_{k=1}^{m} \omega^{k}$ with each $\omega^{k} \in C_{n-1}^{k}$, or there exists $\omega^{\prime} \simeq \omega$ which has an inadmissible subword of length less than or equal to $3 \ell_{n-1}$.

Proof. Suppose that for all $\omega^{\prime} \simeq \omega$, every subword of $\omega^{\prime}$ of length $3 \ell_{n-1}$ is admissible. Let $\ell=s \ell_{n-1}+r$ with $0 \leq r<\ell_{n-1}$. We claim that there exist $\omega^{k} \in M_{n-1}$ and a word $\omega^{*}$ of length $r$ such that $\omega \simeq \prod_{k=1}^{s} \omega^{k} \cdot \omega^{*}$. We will show that $\left(\omega_{i}\right)_{i=1}^{m \ell_{n-1}} \simeq \prod_{k=1}^{m} \omega^{k}$ for all $m \leq s$ by induction on $m$.

Clearly, any admissible word of length $3 \ell_{n-1}$ must include some $a \in M_{n-1}$ as a subword, so $\omega$ must include such a subword. Taking a rotation equivalent of $\omega$ if necessary, we deduce that there exists $\omega^{1} \in M_{n-1}$ such that $\omega_{i}=\omega_{i}^{1}$ for all $1 \leq i \leq \ell_{n-1}$. This proves the case $m=1$.

Given that $\left(\omega_{i}\right)_{i=1}^{m \ell_{n-1}}=\prod_{k=1}^{m} \omega^{k}$ with each $\omega^{k} \in M_{n-1}$, consider the word $b=\left(b_{i}\right)_{i=1}^{2 \ell_{n-1}}$ defined by $b_{i}=\omega_{(m-1) \ell_{n-1}+i}$ for all $1 \leq i \leq 2 \ell_{n-1}$, which is well-defined as long as $m+1 \leq s$. Since $b$ has length $2 \ell_{n-1}<3 \ell_{n-1}$, it is admissible and so there exist $N, M$, $t>0$ and $a=\prod_{k=1}^{M} a^{k} \in M_{N}$ with each $a^{k} \in M_{n-1}$ such that $b_{i}=a_{t+i}$ for all $1 \leq i \leq$ $2 \ell_{n-1}$. By Lemma 3.6 we have $t=\ell_{n-1} \bar{t}$ for some $\bar{t}$, so that $b=a^{\bar{t}+1} \cdot a^{\bar{t}+2}$. Thus $\left(\omega_{i}\right)_{i=m \ell_{n-1}+1}^{(m+1) \ell_{n-1}}=\left(b_{i}\right)_{i=\ell_{n-1}+1}^{2 \ell_{n-1}}=a^{\bar{t}} \in M_{n-1}$ as required to prove the case $m+1$. This completes the induction step and proves our claim. 
Since for any $\omega^{\prime} \simeq \omega$, every subword of $\omega^{\prime}$ with length less than or equal to $3 \ell_{n-1}$ is admissible, the word $z=\omega^{s} \cdot \omega^{*} \cdot \omega^{1}$ must be admissible. By Corollary 3.7 this implies $\ell_{n-1} \mid r$ and hence $r=0$, thus completing the proof.

LEMMA 3.11. Let $\omega=\prod_{k=1}^{m} \omega^{k}$ for some $\omega^{1}, \ldots, \omega^{m} \in M_{n}$ with $m \geq 1$. Then at least one of the following holds:

(a) there exists $\omega^{\prime} \simeq \omega$ such that $\omega^{\prime}$ has an inadmissible subword with length less than or equal to $2 \ell_{n-1} t_{n-1}$;

(b) there exists $\omega^{\prime} \simeq \omega$ such that $\omega^{\prime}$ has an inadmissible subword with length less than or equal to $\left(1+t_{n}\right) \ell_{n}$, and $m \geq q_{n}$; or

(c) there exist $\hat{m}>0$ and $\hat{\omega}^{1}, \ldots, \hat{\omega}^{\hat{m}} \in M_{n+1}$ such that $m=\ell_{n+1} \hat{m}$ and $\omega \simeq \prod_{k=1}^{\hat{m}} \hat{\omega}^{k}$.

Proof. Suppose that case (a) does not hold. Writing $\omega=\prod_{i=1}^{m} c_{j_{i}}^{k_{i}}$ with every $c_{j_{i}}^{k_{i}} \in C_{n}^{k_{i}}$, this assumption implies, via Lemma 3.8(i) and Lemma 3.9, that for every $i<m$ we have $k_{i_{1}}=1+k_{i} \bmod q_{n}$. Clearly, there exists a rotation equivalent of $\omega$ which includes the word $c_{j_{m}}^{k_{m}} \cdot c_{j_{1}}^{k_{1}}$. Again by Lemma 3.8(i) and Lemma 3.9, our assumption that case (a) does not hold implies that this word is admissible, and hence $k_{1}=k_{m}+1 \bmod q_{n}$. It follows that the sequence $k_{i}$ must take every value in the range $1, \ldots, q_{n}$ an equal number of times. Taking a rotation equivalent if necessary, we have

$$
\omega=\prod_{s=1}^{S} \prod_{k=1}^{q_{n}} c_{j_{k, s}}^{k}
$$

for some integer $S \geq 1$ and some sequence $j_{k, s}$. Note in particular that $m \geq q_{n}$ (as required for (b) to hold).

We now suppose also that (b) does not hold. To show that $\omega$ is a concatenation of elements of $M_{n+1}$, and hence that (c) holds, it remains to show that $j_{k, s}=j_{k+t_{n}, s}$ for all $1 \leq s \leq S$ and $1 \leq k \leq q_{n}-t_{n}$. If this is not the case, then $\omega$ must include a subword of the form

$$
\prod_{k=k_{0}}^{k_{0}+t_{n}} c_{j, s}^{k}
$$

for some fixed $s$ such that $j_{k_{0}, s} \neq j_{k_{0}+t_{n}, s}$, which is inadmissible by Lemma 3.8(ii) and has length $\left(1+t_{n}\right) \ell_{n}$, implying the case (b).

Combining the above lemmata, we obtain the following.

Proposition 3.12. Define a set $K \subseteq \Sigma_{A}$ by letting $x \in K$ if and only if every subword of $x$ is admissible. Then $K$ is closed, satisfies $\sigma K=K$, and is non-empty. Moreover,

$$
\sup _{\omega \in \Omega_{p}} \xi(\omega, K)=o\left(p^{\varepsilon}\right)
$$

for every $\varepsilon>0$.

Proof. That $K$ is a non-empty subset of $\Sigma_{A}$ follows from the fact that $M_{n} \subseteq \Omega_{\ell_{n}}$ for every $n \geq 1$. The proof that $K$ is closed and $\sigma K=K$ is straightforward. Given $\omega \in \Omega_{p}$ and $\ell_{n} \leq p<\ell_{n+1}$, we will attempt to bound the quantity

$$
\xi(\omega, K)=\inf _{\omega^{\prime} \simeq \omega}\left\{\ell>0: \exists a \in \Omega_{\ell} \text { such that } a \prec \omega^{\prime} \text { and } a \text { is not admissible }\right\}
$$


Suppose that $\xi(\omega, K)>3 \ell_{n-1}$. Then by Lemma 3.10 we have $\omega \simeq \prod_{i=1}^{m} c_{j_{i}}^{k_{i}}$, where each $c_{j_{i}}^{k_{i}} \in C_{n-1}^{k_{i}}$ and $m=p / \ell_{n-1}$. Lemma 3.11 then implies that either $\xi(\omega, K) \leq 2 \ell_{n-2} t_{n-2}$, or $\xi(\omega, K) \leq \ell_{n-1}\left(1+t_{n-1}\right)$, or $\omega \simeq \prod_{i=1}^{\hat{m}} c_{j_{i}}^{k_{i}}$ where each $c_{j_{i}}^{k_{i}} \in C_{n}^{k_{i}}$ and $\hat{m}=p / \ell_{n}$. In the last of these three alternatives, apply Lemma 3.11 again to see that, since $p<\ell_{n+1}$, either $\xi(\omega, K) \leq 2 \ell_{n-1} t_{n-1}$, or $p \geq \ell_{n} q_{n}$ and $\xi(\omega, K) \leq \ell_{n}\left(1+t_{n}\right) \leq 2 p t_{n} / q_{n}$. We conclude that, in any case,

$$
\begin{aligned}
\xi(\omega, K) & \leq \max \left\{3 \ell_{n-1}, 2 \ell_{n-2} t_{n-2}, \ell_{n-1}\left(t_{n-1}+1\right), 2 \ell_{n-1} t_{n-1}, \frac{2 p t_{n}}{q_{n}}\right\} \\
& =\max \left\{2 \ell_{n-1} t_{n-1}, \frac{2 p t_{n}}{q_{n}}\right\} .
\end{aligned}
$$

Hence, for every $n \geq 3$ and $\varepsilon>0$,

$$
\begin{aligned}
\sup _{\ell_{n} \leq p<\ell_{n+1}} \sup _{\omega \in \Omega_{p}} \frac{1}{p^{\varepsilon}} \xi(\omega, K) & \leq \max \left\{2 \ell_{n-1} t_{n-1} \ell_{n}^{-\varepsilon}, 2 \frac{\ell_{n+1}^{1-\varepsilon} t_{n}}{q_{n}}\right\} \\
& \leq 2 \max \left\{\ell_{n-1}^{1-\varepsilon} t_{n-1} q_{n-1}^{-\varepsilon}, \ell_{n}^{1-\varepsilon} t_{n} q_{n}^{-\varepsilon}\right\}=2 \ell_{n}^{1-\varepsilon} t_{n} q_{n}^{-\varepsilon} .
\end{aligned}
$$

To complete the proof, therefore, it suffices to show that $t_{n} q_{n}^{-\varepsilon} \ell_{n} \rightarrow 0$ for every $\varepsilon>0$. Using Lemma 3.5(vii), Lemma 3.4 and Definition 3.3, we have

$$
\begin{aligned}
\log _{4}\left(t_{n} q_{n}^{-\varepsilon} \ell_{n}\right) & \leq \log _{4}\left(4^{\left(t_{n-1} / \sqrt{\tau_{n}}\right)-\varepsilon t_{n-1}} q_{1} \ell_{1} \prod_{k=2}^{n-1} q_{k}\right) \\
& \leq \log _{4}\left(q_{1} \ell_{1}\right)+\frac{t_{n-1}}{\sqrt{t_{n}}}-\varepsilon t_{n-1}+\sum_{k=1}^{n-2} t_{k} \\
& =-\varepsilon t_{n-1}+\sum_{k=1}^{n-2} t_{k}+O\left(t_{n-1}^{1 / 4}\right) .
\end{aligned}
$$

Elementary analysis then shows that since $t_{n-1} / t_{n}$ tends to zero, $\sum_{k=2}^{n-2} t_{k}=o\left(t_{n-1}\right)$ and so

$$
\log _{4}\left(t_{n} q_{n}^{-\varepsilon} \ell_{n}\right) \rightarrow-\infty
$$

as required.

\section{REFERENCES}

[1] J. Brémont. Gibbs measures at temperature zero. Nonlinearity 16 (2003), 419-426.

[2] T. Bousch. Le poisson n'a pas d'arêtes. Ann. Inst. H. Poincaré Probab. Statist. 36 (2000), 489-508.

[3] T. Bousch and O. Jenkinson. Cohomology classes of dynamically non-negative $C^{k}$ functions. Invent. Math. 148 (2002), 207-217.

[4] T. Bousch and J. Mairesse. Asymptotic height optimization for topical IFS, tetris heaps, and the finiteness conjecture. J. Amer. Math. Soc. 15 (2002), 77-111.

[5] X. Bressaud and A. Quas. Rates of approximation of minimizing measures. Nonlinearity 20 (2007), 845853.

[6] G. Contreras, A. Lopes and P. Thieullen. Lyapunov minimizing measures for expanding maps of the circle. Ergod. Th. \& Dynam. Sys. 5 (2001), 1379-1409. 
[7] B. Hunt and E. Ott. Optimal periodic orbits of chaotic systems occur at low period. Phys. Rev. E 54 (1996), 328-337.

[8] O. Jenkinson. Ergodic optimization. Disc. Cont. Dyn. Syst. 15 (2006), 197-224.

[9] O. Jenkinson. Rotation, entropy and equilibrium states. Trans. Amer. Math. Soc. 353 (2001), 3713-3739.

[10] K. R. Parthasarathy. On the category of ergodic measures. Illinois J. Math. 5 (1961), 648-656.

[11] G. Yuan and B. Hunt. Optimal orbits of hyperbolic systems. Nonlinearity 12(4) (1999), 1207-1224.

\section{CAmbridge JDURNALS}

